\title{
A Real-Time Robust Fall Detection System Using a Wireless Gait Analysis Sensor and an Artificial Neural Network
}

\author{
B.T. Nukala ${ }^{+}$, N. Shibuya ${ }^{+}$, A.I. Rodriguez ${ }^{*}$, J. Tsay ${ }^{+}$, T. Q. Nguyen ${ }^{+*}$, S. Zupancic ${ }^{*}$, and D.Y.C. Lie ${ }^{+*}$ \\ ${ }^{+}$Texas Tech University, Lubbock, Texas, USA \\ *Texas Tech University Health Sciences Center (TTUHSC), Lubbock, Texas, USA
}

\begin{abstract}
This paper describes our custom-designed wireless gait analysis sensor (WGAS) system developed and tested for real-time fall detection. The WGAS is capable of differentiating falls vs. Activities of Daily Living (ADL) and the Dynamic Gait Index (DGI) performed by young volunteers using a Back Propagation Artificial Neural Network (BP ANN) algorithm. The WGAS, which includes a tri-axial accelerometer, 2 gyroscopes, and a MSP430 microcontroller is worn by the subjects at either $\mathrm{T} 4$ (at back) or the belt-clip positions (in front of the waist) for the various falls, ADL, and Dynamic Gait Index (DGI) tests. The raw data is wirelessly transmitted from the WGAS to a nearby PC for real-time fall classification, where six features were extracted for the BP ANN. Overall fall classification accuracies of $97.0 \%$ and $97.4 \%$ have been achieved for the data taken at the $T 4$ and at the belt position, respectively. The preliminary data demonstrates an overall sensitivity of $97.0 \%$ and overall specificity of $97.2 \%$ for this WGAS fall detection system, showing good promise as a real-time low-cost and effective fall detection device for wireless acute care and wireless assisted living.
\end{abstract}

\section{INTRODUCTION}

Approximately one third of the population over 65 years of age fall at least once every year, and the probability of falling increases proportionally with age. Statistics shows that $53 \%$ of senior adults who required hip surgeries related subsequent to a fall will encounter another fall within six months of being released from the hospital [1]. Along with muscle weakness and vision impairment, vestibular disorders are one of the main causes of serious falls in the geriatric population [2]. In a mid-size nursing home with 100 beds, it is estimated that one serious fall will occur every day [1], making it important to be able to wirelessly detect and record falls in both acute care and assisted living settings. For patients with dementia, the need for a robust and easy-to-use real-time automatic fall detection system is relevant as these patients often cannot recall what initiated their fall and the severity of the fall itself. Unless these risk factors are removed, patients will continue to be considered at risk for further falls and injury. Therefore, numerous methods have been proposed to perform automatic fall detection. A skin contact sensor was proposed that used the norms of the tri-axial accelerometer to detect falls [3]. A shock sensor made of a piezoelectric material and a mercury tilt switch was proposed to recognize effects and orientation of the subject [4]. Various radio tags attached to the body were utilized together with machine learning routines for fall recognition [5]. Acceleration vectors generated from the tri-axial accelerometers at trunk and thigh were used in calculating their norms for fall detection [6]. Many fall detection analysis focused around the use of threshold based techniques (i.e., if the signal exceeds the threshold(s) then a fall is detected). However, finding the suitable threshold value(s) that can reliably detect all kinds of falls has proven to be a difficult task, and more sophisticated algorithms are needed to improve the specificity and sensitivity for real-time automatic fall detection systems.

Recently, a few companies have come up with their proprietary fall-detection systems for clinical use, such as [7]:

- Actibelt: uses a 3D-accelerometer hidden in a belt buckle (Trium $\mathrm{GmbH}$ );

- iVi Intelligent Pendant: can detect serious falls and send an alert (Tunstall);

- AutoAlert system with Lifeline: can detect falls and automatically call for help if it detects a fall (Philips);

- PAMSys (Biosensics): gait analysis but not real-time fall detection;

- Shimmer3: provides real-time inertial sensing via accelerometers, gyroscopes, magnetometers and altimeter and with an integrated motion processor for on-board 3D orientation estimation (Realtime Technologies).

We have studied fall detection using our custom sensor without the wireless feature and achieved $100 \%$ accuracy in identifying all 60 falls vs. the activities of daily living (ADL), with no false positives on young volunteers using threshold based algorithms [8]. For the current project, the sensor is wireless and allows for real-time untethered testing for not only fall detections, but also for gait analysis [8,9]. This paper reports our new Wireless Gait Analysis Sensor (WGAS) used specifically for real-time automatic fall detection with an efficient BP ANN algorithm. This WGAS is placed and tested at two different positions on the body: at $\mathrm{T} 4$ and at the belt using a belt clip as shown in Fig. 1. We will show the statistical data with analysis on all fall tests.

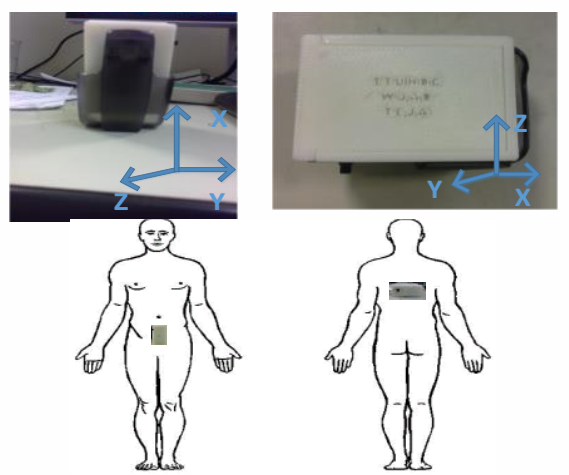

Fig. 1 Sensor orientation and location at Belt position (left) and at T4 (right)

\section{SENSOR SYSTEM AND EXPERIMENTAL METHODS}

\section{A. The Wireless Gait Analysis Sensor (WGAS)}

The custom-designed WGAS consists of a 3-axis linear accelerometer, a single axis gyroscope, and a dual axis gyroscope to measure 3-D human body translations and 
rotations during a gait pattern with the help of these MicroElectrical and Mechanical system (MEMS) sensors integrated on a PCB. This WGAS system is supported by a Texas Instruments (TI) MSP430 microcontroller, and a wireless 2.4 $\mathrm{GHz}$ USB transceiver using the SimpliciTITM protocol with a range of $\sim 12$ meters ( $40 \mathrm{ft}$ ). The 2 AAA batteries used in our earlier wired sensor [7] were replaced by a single rechargeable Li-ion coin battery, providing a battery lifetime of $\sim 48$ hours continuous operation time with each recharge. The PCB, coin battery and the microcontroller are placed in a specially designed $3 \mathrm{D}$ printed box $\left(2.2^{\prime} \times 1.5^{\prime} \times 0.8^{\prime}\right)$ with a total weight of 42 grams. The design of the box was done with a 3D modeling software Rhinoceros (Rhino) and printed using a 3D printer with acrylonitrile butadiene styrene (ABS) plastic. The box has a sliding lid and shown in Fig. 2.

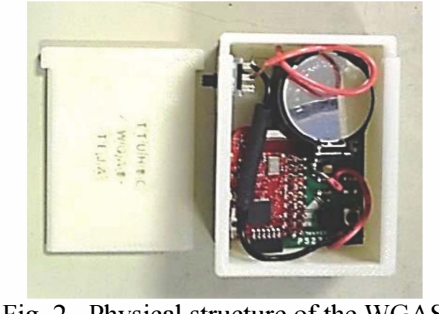

Fig. 2 Physical structure of the WGAS

The accelerometer data is sampled at $160 \mathrm{~Hz}$ and digitized to 8 bits, with its output scaled to $\pm 6 \mathrm{~g}$ at $\Delta V=$ $\pm 6 g / V_{D D}\left(V_{D D}=3.6 \mathrm{~V}\right)$ for each axis. The gyroscope data is also sampled at $160 \mathrm{~Hz}$ and digitized to 8 bits, with its output scaled to $300^{\circ}$ per sec. (dps) at $\Delta V= \pm 300 \mathrm{dps} / \mathrm{So}$ (So (sensitivity) typical value $=0.66 \mathrm{~V} / \mathrm{g}$ for the accelerometer and $3.33 \mathrm{mV} / \mathrm{dps}$ for the gyroscopes). The sensor orientation and positions on the body are shown in Fig. 1. The sensor is carefully secured to the subjects during testing to avoid artifacts. The microcontroller and the transceiver unit enables the real-time transmission of the 6-dimensional gait data wirelessly to the nearby PC, where a LABVIEWTM program is used for designing the Graphical User Interface (GUI) (Fig. $3)$.

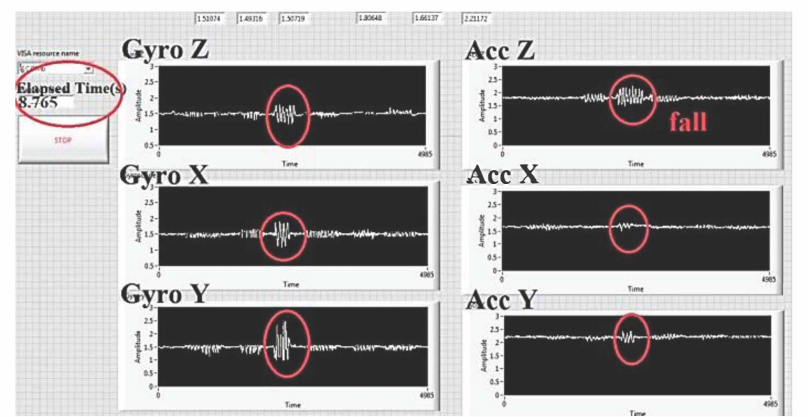

Fig. 3 GUI designed in LABVIEW ${ }^{\text {TM }}$ for our real-time fall detection system using the WGAS, indicating a fall occurred after $8.765 \mathrm{sec}$.

\section{B. Testing of Falls, ADL and DGI}

Intentional falls similar to those reported in [3] were performed on a gymnastics cushioned landing mat (approximately 8" thick) by two young volunteers wearing the WGAS at T4 and on the belt (see Table 1 with 10 types of falls). The total number of falls was 101 (51 at T4 and 50 at belt). For the ADL tests [3], the same volunteers performed every ADL recorded in Table 2 (including picking up an object test \#7), and the aggregate number of ADL was 69 (34 at T4 and 35 at belt). For the DGI tests [10], the same volunteers performed every DGI test in Table 3, with 152 tests ( 82 at $\mathrm{T} 4$ and 70 at belt).

\begin{tabular}{|l|l|}
\multicolumn{1}{c}{ TABLE 1 } & Intentional falls performed in this work [3] \\
\hline Test No. & \multicolumn{1}{c|}{ Description } \\
\hline 1. & Fall back with legs straight \\
\hline 2. & Fall forward with legs straight \\
\hline 3. & Fall back with knees bent \\
\hline 4. & Fall forward with knees bent \\
\hline 5. & Fall left with knees bent \\
\hline 6. & Fall right with knees bent \\
\hline 7. & Fall left with legs straight \\
\hline 8. & Fall right with legs straight \\
\hline 9. & Fall while sitting on a chair \\
\hline 10. & Trip over a small object \\
\hline
\end{tabular}

TABLE 2 Activities of Daily Living (ADL) movements performed [3]
\begin{tabular}{|l|l|}
\hline Test No. & \multicolumn{1}{c|}{ Description } \\
\hline 1. & Sitting down and standing up from an armchair \\
\hline 2. & Sitting down and standing up from a low stool \\
\hline 3. & Sitting down and standing up from a bed \\
\hline 4. & Lying down and standing up from a bed \\
\hline 5. & Walking $10 \mathrm{~m}$ \\
\hline 6. & Stretching while standing \\
\hline 7. & Picking up an object from the floor \\
\hline
\end{tabular}

\begin{tabular}{|l|l|}
\multicolumn{2}{|c}{ TABLE 3 Dynamic Gait Index (DGI) tests performed } \\
\hline No. & \multicolumn{1}{c|}{ Description } \\
\hline 1. & Gait level surface - walk with the normal speed up to 20' mark \\
\hline 2. & $\begin{array}{l}\text { Change in Gait speed- walk with normal pace up to 5'.walk fast } \\
\text { for next 5', walk slowly for next 5' and walk normal for last 5' }\end{array}$ \\
\hline 3. & $\begin{array}{l}\text { Gait with horizontal head turns-walk normal with horizontal } \\
\text { head turns up to 20' mark }\end{array}$ \\
\hline 4. & $\begin{array}{l}\text { Gait with vertical head turns-walk normal with vertical head } \\
\text { turns up to 20' mark }\end{array}$ \\
\hline 5. & $\begin{array}{l}\text { Gait and Pivot turn-walk normal but at the end turn around like } \\
\text { a pivot turn }\end{array}$ \\
\hline 6. & $\begin{array}{l}\text { Step over obstacle-walk normal and when you come across } \\
\text { obstacle step over not around }\end{array}$ \\
\hline 7. & $\begin{array}{l}\text { Step around obstacles-walk normal and when } 1^{\text {st }} \text { obstacle comes } \\
\text { across, walk around right side and when 2nd obstacle comes } \\
\text { across walk around left }\end{array}$ \\
\hline
\end{tabular}

\section{BP ANN ALGORITHM}

Back Propagation Artificial Neural Network (BP ANN) has been applied in numerous areas with excellent results, for example in extraction, classification and prediction [11]. In this paper six simple features ( 2 for each axis) were extracted from the accelerometer and gyroscope sensors for each of the falls, ADL and DGI tests, which are used as the inputs for the classification algorithm as shown in Eqs. 1-2:

Angular velocity Range: $\mathrm{R}_{\omega, x}=\max \left(\omega_{x}\right)-\min \left(\omega_{x}\right) ; \mathrm{R}_{\omega, y}$ $=\max \left(\omega_{y}\right)-\min \left(\omega_{y}\right) ; \mathrm{R}_{\omega, z}=\max \left(\omega_{z}\right)-\min \left(\omega_{z}\right) \ldots(1)$

Acceleration Range: $\mathrm{R}_{a, x}=\max \left(A_{x}\right)-\min \left(A_{x}\right) ; \mathrm{R}_{a, y}$ $=\max \left(A_{y}\right)-\min \left(A_{y}\right) ; \mathrm{R}_{a, z}=\max \left(A_{z}\right)-\min \left(A_{z}\right) \ldots(2)$

\section{A. Back Propagation Artificial Neural Network (BP ANN)}

In the training of the feed-forward ANN classifier, back propagation was applied according to Duda et al. [12]. As described in [13], a 3-layer system was picked as the standard BP ANN. The input layer of the network has six neurons which correspond to the six input feature values. There is one hidden layer holding 10 hidden neurons, which 
number was optimized by adjusting the size of hidden neurons (from one to 15) as shown in Fig. 4, where two output neurons corresponding to the two target classes the network needs to differentiate (the features of all falls are considered as class 1, and all DGI and ADL are as class 2).

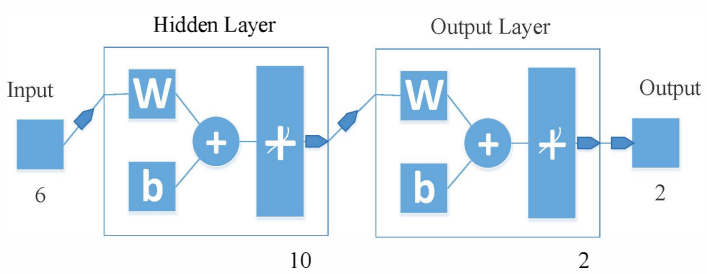

Fig. 4 The 3-layer BP ANN topology used in this work

The multiplication factors of the connections between the layers (or the weightings (W)) are randomly generated between -0.15 to +0.15 . The training, validation and testing of the ANN classifier was performed by dividing the input feature data sets to $70 \%, 15 \%$ and $15 \%$ of its total, respectively. The learning coefficient of the network is adjusted to 0.75 during various experiments until the cross entropy values of the training, validation and testing data sets are small enough (i.e., less than 1).

\section{ClassificATION RESUlTS}

Fall detection using BP ANN was done on WGAS data taken at both $\mathrm{T} 4$ and belt positions. The data was obtained using a $1.7 \mathrm{GHz}$ PC with $4 \mathrm{~GB}$ of RAM, Windows $8 \mathrm{OS}$ and, and the MATLAB R2013b for the BP ANN classifier model. For the trained BP ANN classifier model, the same number of training, validation and testing datasets was used. The BP ANN classifier model learned and converged after 19 epochs for the data from T4 position. The results at T4 were obtained as follows: using the training dataset yielded $96.6 \%$ Correct Detection Rate (CDR), while using testing and validation dataset yielded $100 \%$ and $96.0 \%$ CDRs, respectively. Combining training, testing and validation detection results yielded an overall CDR of $97.0 \%$.

TABLE 4: BP ANN classification results and final parameters from T4 data Parameters

\begin{tabular}{lc} 
Number of Input Neurons & 6 \\
Number of Hidden Neurons & 10 \\
Number of Output Neurons & 2 \\
Learning Coefficient & 0.75 \\
\% of Error & $3 \%$ \\
Number of Epochs & 19 \\
Training* & SCG \\
Gradient & 0.0162 \\
Training optimization time (s) & 0.00 \\
Number of training data set & 117 \\
Number of test data set & 25 \\
Number of Validation data set & 25 \\
Training dataset CDR & $96.6 \%$ \\
Test dataset CDR & $100 \%$ \\
Validation dataset CDR & $96.0 \%$ \\
Overall CDR & $97.0 \%$ \\
\hline
\end{tabular}

*SCG $=$ Scaled Conjugate Gradient

The performance of the BP ANN classifier is determined by the validation cross entropy. Minimizing the cross entropy can give better results. In this case the best validation performance was achieved at epoch 13 with a cross entropy of 0.091.The performance indicators such as specificity and sensitivity were also calculated by using the Eqs. 3-4:

$$
\begin{aligned}
& \text { Specificity }=\frac{T N}{T N+F P} \\
& \text { Sensitivity }=\frac{T P}{T P+F N}
\end{aligned}
$$

where $T N$ (true negatives) are the ADLs and DGIs correctly classified, FP (false positives) are the ADLs and DGIs that were not correctly classified by the BP ANN, TP (true positives) are the falls correctly classified and $F N$ (false negatives) are the falls that were not detected by the BP ANN. The specificity of the BP ANN for fall detection at T4 is $96.5 \%$ and the sensitivity is $98.0 \%$.

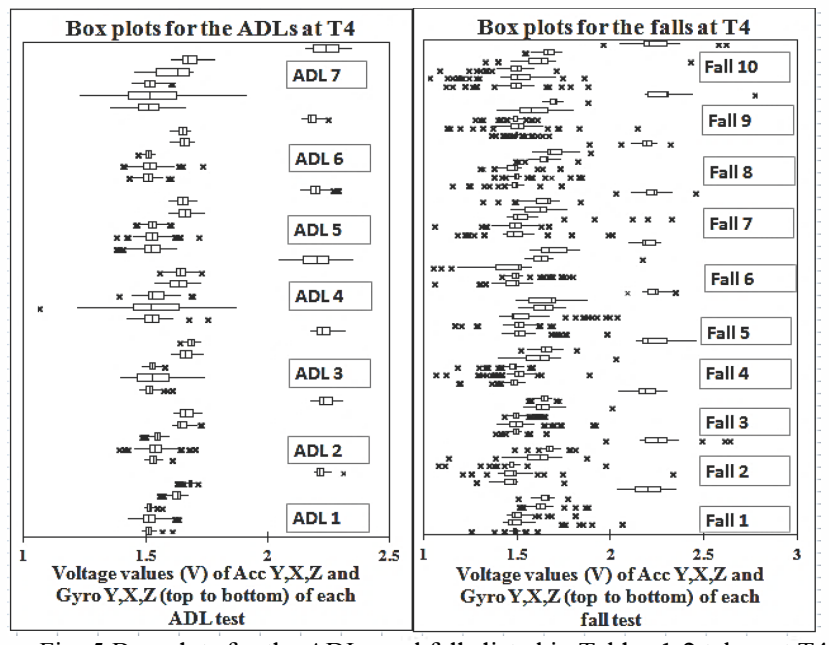

Fig. 5 Box plots for the ADLs and falls listed in Tables 1-2 taken at T4

Fig. 5 shows the box plots for the ADLs vs. falls listed in Table 1 and Table 2 taken at T4. The maximum ranges were recorded for Gyro $\mathrm{X}$ (for $\mathrm{ADL} 4$ and $\mathrm{ADL}$ 7: 0.81V and $0.69 \mathrm{~V}$, respectively), and Acc Y (for ADL 4 and ADL 7: $0.30 \mathrm{~V}$ and $0.19 \mathrm{~V}$, respectively). For the falls, the maximum ranges were recorded for the Gyro $X$ (for Fall 2 and Fall 9: $1.13 \mathrm{~V}$ and $1.02 \mathrm{~V}$, respectively) and Acc Y (for fall 10 and fall 9: $0.66 \mathrm{~V}$ and $0.59 \mathrm{~V}$, respectively). Some more statistics are shown in Table 5 for the T4 data.

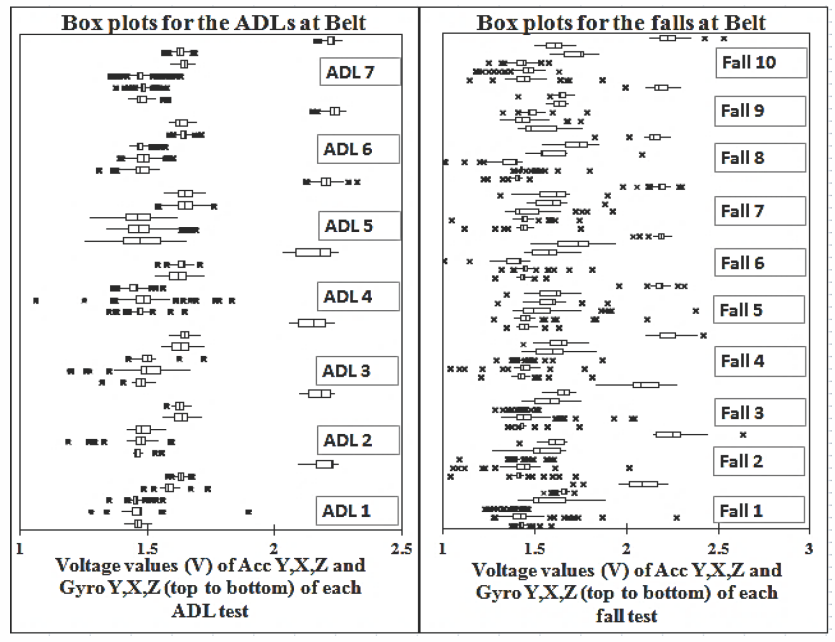

Fig. 6 Box plots for the ADLs and falls listed in Tables 1-2 taken at belt

The BP ANN classifier model learned and converged after 12 epochs for the data taken at the belt position. The results at the belt position were obtained as follows: using the training dataset yielded $98.2 \%$ CDR, while using testing and 
validation dataset yielded $100 \%$ and $91.3 \%$ CDRs, respectively. Combining training, testing and validation detection results yielded an overall CDR of $97.4 \%$, similar to the values obtained for the data taken at $\mathrm{T} 4$.

TABLE 5 Statistical table for ADLs vs. falls at T4

\begin{tabular}{|c|c|c|c|c|}
\hline Stats & $\begin{array}{c}\text { Gyro X } \\
\text { (ADL 4) }\end{array}$ & $\begin{array}{c}\text { Acc Y } \\
\text { (ADL 4) }\end{array}$ & $\begin{array}{c}\text { Gyro X } \\
\text { (fall 2) }\end{array}$ & $\begin{array}{c}\text { Acc Y } \\
\text { (fall 10) }\end{array}$ \\
\hline Mean & 1.532 & 2.201 & 1.482 & 2.234 \\
\hline STDEV & 0.134 & 0.071 & 0.153 & 0.101 \\
\hline Min. & 1.062 & 2.050 & 1.206 & 1.956 \\
\hline Median & 1.522 & 2.205 & 1.479 & 2.208 \\
\hline Max. & 1.871 & 2.352 & 2.331 & 2.616 \\
\hline Range & 0.81 & 0.302 & 1.125 & 0.66 \\
\hline
\end{tabular}

Fig.6 shows the box plots for ADLs vs. falls taken at belt. The maximum ranges were recorded for Gyro X (for ADL 4 and ADL 1: 077V/0.6V, respectively) and Acc Y (for ADL 4 and $\mathrm{ADL}$ 3: $0.21 \mathrm{~V} / 0.17 \mathrm{~V}$, respectively). For falls, the maximum ranges were recorded for Gyro $X$ (for Fall 2 and Fall 1: $0.96 \mathrm{~V} / 0.92 \mathrm{~V}$, respectively) and Acc X (for Fall 7: $0.59 \mathrm{~V}$ ), as shown in Table 6 . The specificity of the BP ANN for falls detection at belt is $98.1 \%$ and sensitivity is $96.0 \%$. In this case the best validation performance was achieved at epoch 6 with a cross entropy of 0.283 .

TABLE 6 Statistical table for ADLs vs. falls at belt

\begin{tabular}{|c|c|c|c|c|}
\hline Stats & $\begin{array}{c}\text { Gyro X } \\
\text { (ADL 4) }\end{array}$ & $\begin{array}{c}\text { Acc Y } \\
\text { (ADL 4) }\end{array}$ & $\begin{array}{c}\text { Gyro X } \\
\text { (fall 2) }\end{array}$ & $\begin{array}{c}\text { Acc X } \\
\text { (fall 7) }\end{array}$ \\
\hline Mean & 1.495 & 2.151 & 1.431 & 1.591 \\
\hline STDEV & 0.088 & 0.068 & 0.114 & 0.093 \\
\hline Min. & 1.059 & 2.033 & 1.044 & 1.306 \\
\hline Median & 1.487 & 2.179 & 1.446 & 1.617 \\
\hline Max. & 1.830 & 2.246 & 2.008 & 1.892 \\
\hline Range & 0.77 & 0.213 & 0.964 & 0.586 \\
\hline
\end{tabular}

TABLE 7 BP ANN classification results and final parameters (data at belt)

\section{Parameters}

Number of Input Neurons

Number of Hidden Neurons

Number of Output Neurons

Learning Coefficient

$\%$ of Error

Number of Epochs

Training*

Gradient

Training optimization time (s)

Number of training dataset $\quad 109$

Number of test dataset 23

Number of Validation data set $\quad 23$

Training dataset CDR $\quad 98.2 \%$

Test dataset CDR $\quad 100 \%$

Validation dataset CDR $\quad 91.3 \%$

Overall CDR $97.4 \%$

* $\mathrm{SCG}=$ Scaled Conjugate Gradient

Table 8 shows the results of this work compared with previously published work in the literature. Please note we did not include [3] in Table 8 because the testing subjects for falls vs. ADL were not from the same group. Many reported studies $[6,11,15]$ uses a single 3-axial accelerometer to detect falls; also threshold based algorithms proved to be complicated yet they can be successful for fall detection. Our WGAS uses both accelerometer and gyroscopes for real-time analysis of falls by using BP ANN algorithm is robust in detecting falls with high accuracy and sensitivity in $<0.01 \mathrm{~s}$. Moreover, the BP ANN algorithm can also be implemented in LABVIEW ${ }^{\mathrm{TM}}$ next to streamline and speed up real time detection. Future work includes conducting more fall and ADL tests on geriatric and high-risk patients to improve clinical usage of this fall detection system, and also in parallel for gait analysis on patients with balance disorders for fall prevention (IRB already in-place and going through clinical trials now). We will also explore the more advanced classifier such as Support Vector Machine (SVM).

TABLE 8 Our real-time WGAS fall-detection system vs. the literature

\begin{tabular}{|l|l|l|l|}
\hline & Sensors & Sensitivity & Specificity \\
\hline$[14]$ & 1 Acc, 2 Gyro & 91.0 & $92.0 \%$ \\
\hline$[15]$ & 1 Acc & $80.0 \%$ & $88.0 \%$ \\
\hline$[6]$ & 1 Acc & $94.0 \%$ & $94.0 \%$ \\
\hline$[11]$ & 1 Acc & $98.4 \%$ & $98.6 \%$ \\
\hline This & 1 Acc., 2 Gyro & $98.0 \%$ (at T4) & $96.5 \%$ (at T4) \\
work & & $96.0 \%$ (at belt) & $98.1 \%$ (at belt) \\
\hline
\end{tabular}

\section{CONCLUSION}

Our custom wireless gait analysis sensor (WGAS) was applied for real-time automatic fall detection with a simple but very fast ANN with 6 input features trained by back propagation. The results show that falls are automatically detected at both $\mathrm{T} 4$ and belt positions with an overall sensitivity of $97.0 \%$ and specificity of $97.2 \%$ (i.e., 98 out of 101 falls correctly classified at both positions and 215 out of 221 ADL+DGI tests correctly classified). The preliminary data on young volunteers suggests our real-time fall detection system performs competitively compared to other systems, whether placed at T4 or on a waist-level belt. For example, Table 8 shows the best accuracy of $\sim 98.5 \%$ was reported in [11]; however in that work various types of falls were not tested so its actual specificity is expected to be lower. In addition, for the falls that show small acceleration, gyroscopes that can pick up noticeable angular changes are needed to detect these falls (e.g., slow falling from a chair as fall \#9 of Table 1), Our fall detection system using both accelerometers and gyroscopes can be superior to systems using accelerometers only, and further improvement on our classifiers design and performance are also underway.

\section{REFERENCES}

[1] Centers for Disease Control and Prevention, http://www.cdc.gov/homeandrecreationalsafety/falls/adultfalls.html

[2] G. F. Fuller, American Family Physician, vol. 61, no. 7, pp. 2159-68, 2173-4, Apr 1, 2000

[3] Ravi Narasimhan, Proc. IEEE Int'l EMBS Conf., pp. 4038-41, 2012

[4] G. Williams et al., Proc. IEEE Int'l EMBS Conf., pp. 1151-57, 1998

[5] M. Lustrek and B. Kaluza, Informatica, 33, 3, pp. 205-212, 2009

[6] A. K. Bourke et al., J. of Biomechanics. Vol. 43, pp. 3051-57 2010

[7] S. Patel et al., Abs. $7^{\text {th }}$ World Congress of Biomechanics, Boston, July 6-11, 2014

[8] J. Jacob et al., Proc. IEEE Int'l Conf. on Fuzzy Logics, pp. 666-671, 2011

[9] B.T. Nukala et al., Actas. IEEE Conf. on Information Systems and Techno. (CISTI), pp. 160-161, 2014

[10] K. Perrel et al., J. Gerontology Series A - Biological Sciences \& Medical Sciences. 56 (12): M761-766. 2001

[11] M. Vallejo et al., Proc. IEEE Int'l EMBS Conf., pp.1648-51, 2013

[12] R.O. Duda et al., Pattern Classification, New York: WileyInterscience; 2001

[13] S. Dreiseitl et al., J Biomed Inform, 35:352-9 2002

[14] Q. Li, et al., Proc. IEEE $6^{\text {th }}$ Int'l Workshop on Wearable and Implantable Body Sensor Networks (BSN), pp. 138-143. 2009

[15] J. T. Perry et al., Proc. IEEE 6th Int'l Symp. on High-Capacity Optical Networks and Enabling Technologies (HONET), pp. 158-64, 2009 